\title{
Orientational order in xenon fluid monolayers on single crystals of exfoliated graphite
}

\author{
S. E. Nagler* and P. M. Horn \\ IBM Thomas J. Watson Research Center, Yorktown Heights, New York 10598
}

T. F. Rosenbaum

The James Franck Institute and Department of Physics, University of Chicago, Chicago, Illinois 60637

R. J. Birgeneau, M. Sutton, and S. G. J. Mochrie

Department of Physics, Massachusetts Institute of Technology, Cambridge, Massachusetts 02139

D. E. Moncton

Physics Department, Brookhaven National Laboratories, Upton, New York 11973

Roy Clarke

Department of Physics, The University of Michigan, Ann Arbor, Michigan 48109

(Received 3 December 1984)

\begin{abstract}
The melting transition of Xe monolayers adsorbed on a single-crystal exfoliated graphite substrate has been studied by high-resolution synchrotron $x$-ray scattering. At temperatures slightly above the melting transition the fluid phase has a high degree of orientational order. The results are discussed in the context of current theories of two-dimensional melting including the effects of the substrate.
\end{abstract}

\section{INTRODUCTION}

The nature of the melting transition in simple twodimensional (2D) systems remains an intriguing and unresolved problem. In this paper we report a synchrotron $\mathrm{x}$-ray scattering study of correlations in a monolayer of Xe physisorbed on the basal planes of a single-crystal exfoliated graphite substrate. ${ }^{1,2}$ As we will discuss below, the incommensurate $\mathrm{Xe}$ monolayer is an excellent realization of a simple 2D solid; the principal effect of the substrate is to impose an effective orientational ordering field $h_{6}$ on the monolayer. ${ }^{3}$

The structure and phase transitions of rare-gas monolayers physisorbed on graphite have been studied extensively with a variety of techniques including thermodynamic measurements, ${ }^{4}$ low-energy electron diffraction ${ }^{5}$ neutron scattering, ${ }^{6}$ and x-ray diffraction. ${ }^{7}$ Much of this work has been summarized by Heiney et al. ${ }^{8}$ and Dimon et al. ${ }^{9}$ The melting of $\mathrm{Xe}$ monolayers on $Z Y X$ exfoliated graphite has been investigated by high-resolution $x$-ray scattering using synchrotron radiation., 8 The earlier experiments demonstrated that at coverages somewhat below one monolayer the melting is first order, but near and above one monolayer the transition is continuous. It has been conjectured that a tricritical point separates the two regions. $4,8,9$

In most of the previous $x$-ray structural studies, the basal planes of the substrate were composed of randomly oriented crystallites. This precluded the possibility of investigating orientational effects in the correlations. In the present work, the single-crystal nature of the substrate has allowed us to probe the behavior of the orientational correlations and to address important questions regarding the existence of the hexatic phase ${ }^{10,11}$ and the effect of the substrate on the transition. The present work is an extension of, an improvement upon, a previous experiment using a rotating-anode $x$-ray source and a lower-resolution scattering configuration. ${ }^{2}$

Section II of this paper contains a brief discussion of current theories of melting in two dimensions. Our experimental procedure and data analysis is described in Sec. III. The results of the experiment and the nature of the orientationally ordered fluid state are discussed in Sec. IV.

\section{THEORY OF MELTING IN TWO DIMENSIONS}

The two-dimensional melting transition is an example of a finite-temperature, lower-marginal-dimensionality phase transition. Kosterlitz and Thouless ${ }^{12}$ and Berezinskii $^{13}$ popularized the modern description of this type of transition by explicitly proving that the 2D $X Y$ model, and symmetry-equivalent models, can have topological defects which mediate phase transitions. Examples of topological defects include vortices in a 2D superfluid or superconductor, magnetic vortices in the 2D $X Y$ model, and dislocations in the $2 \mathrm{D}$ solid. In the low-temperature phase, only bound pairs of thermally generated defects are thermodynamically stable. At the critical temperature $T_{M}$ the defects begin to unbind. For $T>T_{M}$ there is a finite density of unbound defects causing an exponential decay of order with the characteristic length being the average separation between unbound defects.

Defect-mediated transitions can be crudely divided into two generic classes. Transitions in the 2D $X Y$ model, superfluid, superconductor, Coulomb gas, and roughening (solid-on-solid) model belong to one class. ${ }^{14}$ This class of problems is characterized by a scalar interaction between defects. In general, these problems contain no cubic in- 
TABLE I. Correlation functions at long wavelengths.

\begin{tabular}{lll}
\hline \hline Temperature & Positional correlations & Orientational correlation \\
\hline$T<T_{M}$ (solid) & $\begin{array}{l}\langle\rho(0) \rho(r)\rangle \sim 1 / r^{\eta} \\
\text { (power-law decay) }\end{array}$ & $\langle\theta(0) \theta(r)\rangle$ ordered \\
$T_{M}$ & dislocation unbinding & \\
$T_{M<T<T_{H M} \text { (hexatic) }}$ & $\langle\rho(0) \rho(r)\rangle \sim e^{-\kappa r}$ & \\
& (exponential decay) & $\left\langle(\theta(0) \theta(r)\rangle \sim 1 / r^{\eta_{6}}\right.$ \\
$T_{H M}$ & disclination unbinding & (power-law decay) \\
$T>T_{H M}$ (liquid) & $\langle\rho(0) \rho(r)\rangle \sim e^{-\kappa r}$ & \\
& (exponential decay) & $\langle\theta(0) \theta(r)\rangle \sim e^{-\kappa^{\prime} r}$ \\
\hline \hline
\end{tabular}

variant in the coarse-grained microscopic Hamiltonian and the transition in three dimensions is continuous (except that $T_{M}$ can be zero or infinite). As a class, the scalar problems are on particularly strong theoretical ground, and there is good experimental evidence that the Kosterlitz-Thouless (KT) mechanism is operative. ${ }^{14}$

The second class of problems is characterized by a vector interaction between defects. Examples include the two-dimensional melting transition, the "Laplacian roughening model," its dual, the vector Coulomb gas, ${ }^{15,16}$ and the three-dimensional (3D) nematic-smectic $A$-smectic $C$ multicritical point. ${ }^{17}$ These systems are particularly interesting in that the vector nature of the defect-defect interaction precludes all order being lost at a single continuous transition.

The ideas of Kosterlitz and Thouless have been extended to describe the vector problems (including substratefree 2D melting) by Halperin and Nelson ${ }^{10}$ and by Young $^{11}$ (referred to in what follows as the KTHNY theory). The essential features of the theory are that melting occurs in two steps, each associated with a continuous "KT-type" phase transition. As the 2D solid is heated, dislocation pairs unbind at a temperature $T_{M}$, resulting in a phase with orientational order only (the "hexatic" phase). At a higher temperature, $T_{H M}$, the unbinding of disclinations leads to the usual isotropic liquid phase. The behavior of the long-wavelength positional and orientational correlation functions in each phase is summarized in Table I. As discussed below, the presence of a substrate can substantially alter this picture.

If the KTHNY theory outlined above does describe 2D melting, it is likely to be valid only in the limit of a low density of dislocations at the unbinding temperature $T_{M}$. It this condition is not fulfilled, another mechanism, such as the spontaneous generation of grain boundaries, might drive the melting transition. Chui ${ }^{18}$ finds that melting in the absence of a substrate always proceeds by a grainboundary mechanism and is inherently first order. Kleinert ${ }^{19}$ finds that the presence of free disclinations will lead to a first-order melting transition. These findings may be contrasted with that of Marchenko ${ }^{20}$ who claims that first-order phase transitions cannot occur in $2 \mathrm{D}$ systems.

The 2D melting transition of an adsorbed monolayer can be strongly modified by the substrate. Halperin and
Nelson ${ }^{10}$ considered these effects in detail. For an incommensurate solid on a hexagonal substrate, the major effect is to generate a sixfold orientational aligning field $h_{6}$. This field is expected to smear out the hexatic-to-liquid disclination-unbinding transition. Furthermore, if the transition in the absence of a substrate was inherently first order, a nonzero $h_{6}$ may suppress orientational fluctuations enough to cause a continuous transition. Chui ${ }^{18}$ has speculated that a finite $h_{6}$ can suppress the grainboundary melting mechanism and may make the dislocation unbinding mechanism relatively more important. More will be said about this later.

The analytic theories of melting have been complemented by many computer simulations. These have been reviewed recently by Morf. ${ }^{21}$ Direct simulations of xenon on graphite by $\mathrm{Abrham}^{22}$ and co-workers have produced results that seem to agree well with some features of $x$-ray scattering experiments ${ }^{2,8,9}$ but which they interpret as evidence for a first-order melting transition. The direct simulations of real systems are difficult largely because of the long times necessary to achieve equilibrium. To circumvent this problem there have been a number of simulations of model systems.

Saito $^{23}$ directly modeled a system of dislocations and found that for small dislocation core energies $E_{c}$ the melting transition is first order, while for sufficiently large $E_{c}$ the transition is continuous. Simulations of the Laplacian roughening model ${ }^{16}$ have found a continuous transition with evidence for the presence of a hexatic phase. Morf $^{21,24}$ has investigated the melting of a $2 \mathrm{D}$ electron system in the presence of a nonzero $h_{6}$ and concluded that for $h_{6}$ greater than some unknown critical value $h_{6}^{*}$ melting appears to be described by the KTHNY theory. For $h_{6}<h_{6}^{*}$ his system does not equilibrate.

It should be clear from the preceding discussion that the two-dimensional melting problem is far from being solved. We should emphasize that the feature that makes this class of problems unique and controversial is the potential existence of an intermediate phase with orientational order. It is exactly this feature which can now be addressed with exfoliated single-crystal substrates and which is the subject of this paper. In a subsequent section of this paper we discuss the relationship of these theories and the effect of $h_{6}$ to our results from x-ray studies of Xe melting. 


\section{EXPERIMENTAL DETAILS AND DATA ANALYSIS}

The single crystal exfoliated graphite was the same specimen used in the previous low-resolution x-ray experiment; ${ }^{2}$ the method of sample preparation has been described elsewhere. ${ }^{1}$ The effective surface area of the crystal was estimated as roughly 2 Torr $\mathrm{cm}^{3}$ from the $B$ point of a krypton isotherm at $80 \mathrm{~K}$. This compares with surface areas of $100-600$ Torr $\mathrm{cm}^{3}$ for typical $Z Y X$ exfoliated graphite samples used in other $x$-ray experiments. ${ }^{8,9}$

Immediately before the experiment the graphite crystal was cleaned by baking it at $1000^{\circ} \mathrm{C}$ for 30 min under a vacuum of $\sim 10^{-6}$ Torr. The crystal was mounted in a beryllium-window sample cell attached to the cold finger of a displex closed-cycle refrigerator. A stainless-steel bellows connected the cell to an external gas-handling system identical to that used in experiments on $Z Y X$ graphite. $8,9,25$

The cryostat was mounted on the six-circle Huber gonimoter at beam line VII-2 at the Standford Synchrotron Radiation Laboratory (SSRL). The instrumentation and resolution conditions of this beam line have been discussed by Moncton and Brown. ${ }^{26}$ The x-ray wavelength of $1.742 \AA$ was selected by a double-asymmetric-cut $\mathrm{Ge}(111)$ monochromator, and the beam was passed through a monitor so that all data points were collected for a fixed number of incident photons. The beam scattered from the specimen was analyzed by a Ge(111) crystal aligned in the nondispersive configuration and detected by a $\mathrm{NaI}$ scintillation counter.

The scattering from adsorbed monolayers was measured in transmission with the graphite crystal oriented in the ( $h k 0)$ zone so that the $c$ axis was perpendicular to the scattering plane. Under these conditions, the longitudinal resolution near the $\mathrm{Xe}(10)$ peak is $\sim 3 \times 10^{-4} \AA^{-1}$ [halfwidth at half maximum (HWHM)] in the scattering plane and the out-of-plane resolution is $\sim 3 \times 10^{-2} \AA^{-1}$.

The structure of the substrate was characterized first in the absence of adsorbed gas. The mosaic spread of the graphite $(002)$ peak was measured prior to the experiment using $\mathrm{Cu} K \alpha$ radiation from a rotating-anode $\mathrm{x}$-ray source and found to be described by a Lorentzian with a HWHM of $16^{\circ}$. The crystal was further characterized at SSRL as shown in Fig. 1. The lower panel shows radial and angular scans (rocking curves) of the graphite(100) Bragg peak. The mosaic spread of the substrate in the basal plane is fitted to a Lorentzian, shown by the solid line in panel (d), with a HWHM of $2.3^{\circ}$.

The upper panel shows scattering from an adsorbed monolayer of $\mathrm{Kr}$ in the commensurate $\sqrt{3} \times \sqrt{3}$ solid phase. $^{27}$ In panel (a) the solid line shows a fit of the radial scan (see Fig. 3) to a finite-size-broadened $\delta$ function using a method described below. The line shape is assumed to be a Gaussian distribution, $S(Q) \propto e^{-q^{2} L^{2} / 4 \pi}$, where $\mathbf{q}=\mathbf{Q}-\mathbf{G}$ is the difference of the wave vector from a reciprocal-lattice vector and $L$ is the average size of a coherent crystalline domain. The fitted domain size is $\sim 1400 \AA$, consistent with domain sizes found in $Z Y X$ substrates.

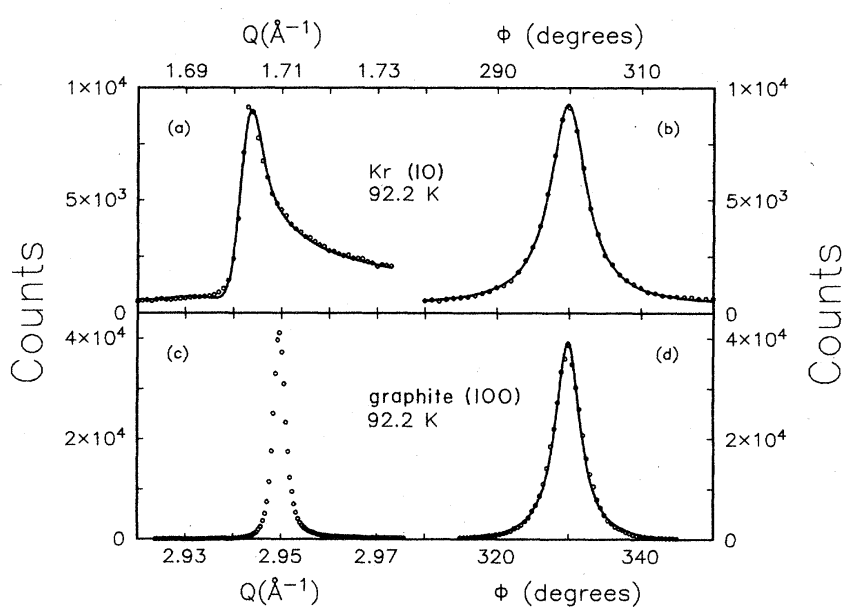

FIG. 1. Scans characterizing the single-crystal exfoliated graphite substrate. Panels (a) and (b) are, respectively, radial and angular scans through the finite-size-limited Bragg peak of a commensurate $\mathrm{Kr}$ solid monolayer. The asymmetric nature of the radial peak (a) is characteristic of two-dimensional scattering. The bottom panels (c) and (d) show corresponding scans through the graphite (100) Bragg peaks. The vertical scale for curves (a) and (b) is counts per $10^{5}$ monitor counts, while for curves $(\mathrm{c})$ and $(\mathrm{d})$ it is counts per $10^{4}$ monitor counts.

The intensity of the $\mathrm{Kr}$ peak is approximately 9000 counts above a background of roughly 500 counts per $10^{5}$ monitor counts $(\sim 6 \mathrm{sec})$. This count rate is comparable to peak intensities observed for overlayers on $Z Y X$ substrates. Thus, the much smaller effective surface area of the exfoliated single crystal is compensated for by the greatly improved in-plane mosaic. The upper right-hand panel shows the corresponding angular scan through the $\mathrm{Kr}$ peak. The rocking curve is noticeably broader than the graphite (100) curve and is described by a Lorentzian of HWHM $3.2^{\circ}$ shown by the solid line. In the commensurate solid phase the $\mathrm{Kr}$ lattice corresponds directly with the available set of adsorption sites of the graphite basal plane. The extra angular width of the $\mathrm{Kr}$ peak relative to the graphite $(100)$ peak suggests that the available surface of the graphite that can accommodate adsorbed gases has a broader mosaic spread than the bulk graphite crystal. Thus, an ideal two-dimensional solid overlayer with perfect orientational long-range order is expected to have a mosaic spread of $3.2^{\circ}$ HWHM in our sample. In the analysis that follows, we use the $3.2^{\circ} \mathrm{HWHM}$ of the krypton rocking curve as the effective substrate mosaic.

We now describe the xenon melting measurements and data analysis. The scans were obtained in a closed-cell configuration: A known amount of $\mathrm{Xe}$ was admitted to the sample cell, the cell was sealed off, and data points were collected at various temperatures in the vicinity of the melting transition. Two sets of data were taken. In the first, approximately 4.2 Torr $\mathrm{cm}^{3}$ of gas was admitted to the cell, and melting occurred at a temperature of $T \sim 138 \mathrm{~K}$ with the xenon-gas pressure in the cell estimated to be about 1 Torr. At this point on the phase diagram (shown schematically in Fig. 2) melting has previously been shown to be continuous in experiments on $Z Y X$ graphite. ${ }^{8,9}$ A second (less extensive) investigation was performed at $T \sim 116 \mathrm{~K}$, where the melting was previously 


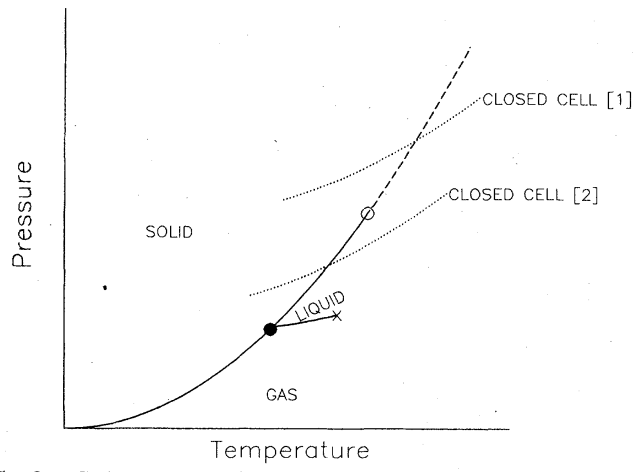

FIG. 2. Schematic phase diagram of Xe on graphite in the monolayer region. The dotted lines show the temperature scans of the present work. The melting transition has been previously found to be first order (solid line) for $T<T_{2}$ and continuous (dotted line) for $T>T_{2}$.

found to be first order.

At each temperature the $\mathrm{Xe}(10)$ peak was characterized by making in-plane radial and angular scans as illustrated in Fig. 3. We note that the angular scans are simply rocking curves through the peak position. Model structure factors were then fitted to the measured profiles, properly accounting for the background and effects of sample mosaic spread.

The background was measured with no gas in the sample cell at temperatures of 115 and $200 \mathrm{~K}$. In the neighborhood of the $\mathrm{Xe}(10)$ peak, the background is dominated by graphite thermal diffuse scattering, and is smooth except for a powder peak at $1.62 \AA^{-1}$ thought to arise from a Sb contaminant. ${ }^{1}$ The change in graphite thermal diffuse scattering as a function of temperature was estimated by linear interpolation and found to vary by less than 5 parts in $10^{4}$ per degree Kelvin over the range of momentum transfer $1.3-1.8 \AA^{-1}$. The background was also measured at $200 \mathrm{~K}$, with 10 Torr of Xe gas (vapor only) in the cell, and at $160 \mathrm{~K}$, with 0.03 Torr Xe present, in order to measure the scattering from the Xe vapor. The gas scattering was found to be flat over the range 1.3-1.8 $\AA^{-1}$ with a magnitude of $\sim 2.7$ counts per $10^{6}$ monitor counts for each Torr $\mathrm{cm}^{3}$ of $\mathrm{Xe}$ in the sample cell.

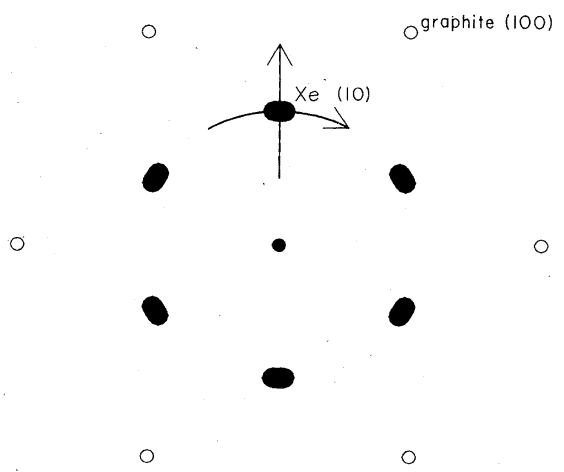

FIG. 3. Reciprocal space of the Xe-on-graphite system. The open circles are graphite Bragg peaks in the basal ( $h k 0)$ plane. The solid distributions represent $\mathrm{Xe}$ diffuse scattering. The directions of radial and angular scans are indicated by the arrows.
A simple calculation shows that attenuation of the scattering arising from x-ray absorption by the adsorbed Xe layer is expected to be negligible. This was confirmed in practice by measuring the intensity of the graphite(100) peak with and without adsorbed Xe present, and also by allowing the absorption coefficient to be a variable parameter in the fits and thereby confirming that there was no systematic statistical improvement. $X$-ray absorption was important in the experiments on $Z Y X$ graphite g,9 $^{8}$ because of the much higher specific surface areas of the substrate.

The scattering intensity at a momentum transfer $\mathbf{Q}$ can be written

$I(\mathbf{Q})=B(\mathbf{Q})+\int R\left(\mathbf{Q}-\mathbf{Q}^{\prime}\right)\left|f\left(\mathbf{Q}^{\prime}\right)\right|^{2}\left\langle S\left(\mathbf{Q}^{\prime}\right)\right\rangle d Q^{\prime}$,

where $B(\mathbf{Q})$ is the measured background, $f(\mathbf{Q})$ is the $\mathrm{Xe}$ form factor, $\langle S(\mathbf{Q})\rangle$ is the structure factor averaged over the orientational distribution of the crystallites, and $R\left(\mathbf{Q}-\mathbf{Q}^{\prime}\right)$ is the instrumental resolution function. In practice, for the present experiment, the resolution can be considered as a perfect $\delta$ function since the finite-size broadening introduced by the average domain of $\sim 1400$ $A$ is an order of magnitude larger than the instrumental resolution width.

A typical $\mathrm{Xe}(10)$ incommensurate-solid line shape is shown in Fig. 4. The solid lines are the results of leastsquares fits to the data. The background, and the vertical and transverse mosaics, are fixed at their measured and known values, respectively, in the analysis of this and all subsequent data. The fitted radial line shape is a finitesize-broadened power law, ${ }^{28}$ given by the Kummer function $^{29}$ (degenerate hypergeometric function)

$$
S(\mathbf{q})=A \Phi\left[1-\frac{\eta}{2} ; 1 ; \frac{-q^{2} L^{2}}{4 \pi}\right],
$$

where $A$ is a scaling factor, $\mathbf{q}=\mathbf{Q}-\mathbf{G}$ (these are now vectors in two dimensions), $L$ is the domain size, and $\Phi(a ; b ; c)$ is the Kummer function. The parameters allowed to vary are $A, G$, and $\eta$. If $L$ is allowed to vary, the best fit occurs when $L \sim 1420 \AA$, consistent with the result from the $\mathrm{Kr}$ commensurate peak. The domain size is fixed at this value for other fits in the solid phase.

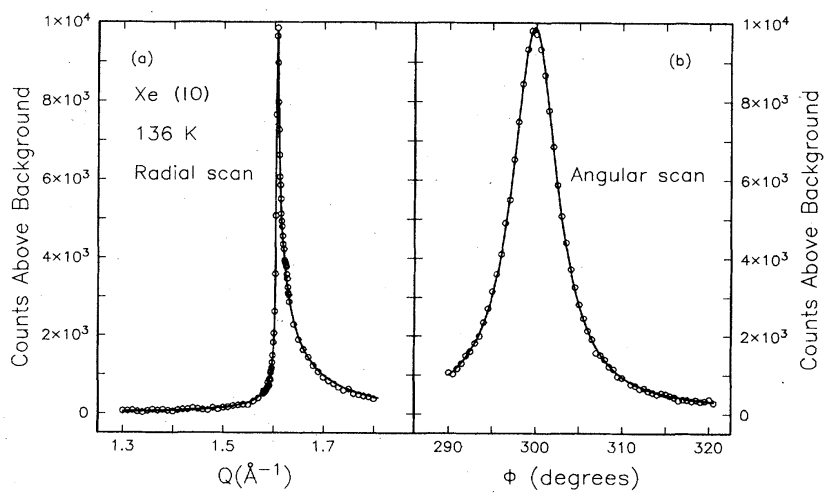

FIG. 4. Xe incommensurate solid peak. The radial scan (a) and angular scan (b) were measured with $1 \times 10^{5}$ monitor counts per point. The measured background has been subtracted from the points shown. The solid line in (a) is a fitted finite-sizebroadened power law with $\eta=0.21$. The solid line in (b) is Lorentzian fit as described in the text. 
The angular scan is fitted to a Lorentzian in angle with the peak position, width, and an overall amplitude allowed to vary. The fitted HWHM is $3.2^{\circ}$, consistent with the measured mosaic width of a commensurate $\mathrm{Kr}$ peak. The large mosaic spread of the sample does not allow us to investigate the possibility of an anisotropic power-law line shape that has been predicted ${ }^{30}$ for a $2 \mathrm{D}$ solid.

The radial scans in the vicinity of the melting transition at $T \sim 138 \mathrm{~K}$ were fitted by assuming three model line shapes for the full two-dimensional scattering: the power-law-decay solid given by Eq. (2), a symmetric Lorentzian spot

$$
S(\mathbf{Q})=A\left[(\mathbf{Q}-\mathbf{G})^{2}+\kappa^{2}\right]^{-1},
$$

and a Lorentzian ring depending only on $Q \equiv|\mathbf{Q}|$,

$$
S(Q)=A\left[(Q-G)^{2}+\kappa^{2}\right]^{-1} \text {. }
$$

Three parameters were allowed to vary for each fit: the peak position $G$, amplitude $A$, and inverse correlation length $\kappa$.

For a system with perfect orientational order, the full two-dimensional line shape should be a circular Lorentzian spot. As the orientational fluctuations increase, the scattering should broaden in the angular direction. The Lorentzian-spot and Lorentzian-ring functional forms represent the extreme symmetric and asymmetric limits, respectively. ${ }^{8,9}$ When the intrinsic transverse width of the scattering is much smaller than the substrate mosaic, i.e., in a well-correlated state, it is expected that the Lorentzian spot provides an adequate approximate line shape. In the limit where the transverse widths greatly exceed the mosaic spread, i.e., in a liquid state with, at most, weak orientational order, the Lorentzian ring is expected to describe the actual scattering profile. In the absence of detailed theoretical characterization of the total line shape, we have chosen these simple forms for purposes of calculational simplicity and straightforward interpretation of the data. It is our belief that within $\sim 2 \mathrm{~K}$ of the melting temperature, the Lorentzian-spot line shape describes the data well enough to allow extraction of the radial correlation lengths. Furthermore, the different line

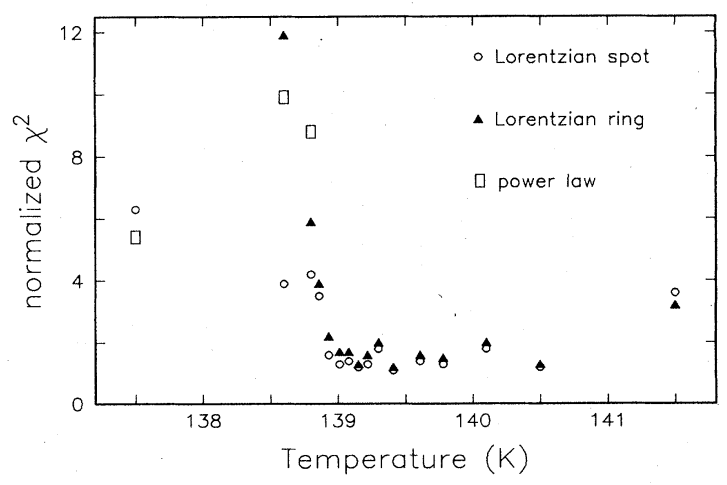

FIG. 5. Normalized $\chi^{2}$ for model line-shape fits of radial scans. A selection of points is shown. At high temperatures the $\kappa^{2}$ for power-law fits (rectangles) diverge; the same is true for the Lorentzian-ring fits (triangles) at low temperatures. The Lorentzian-spot line shape (open circles) provides a reasonable fit over the entire temperature range.

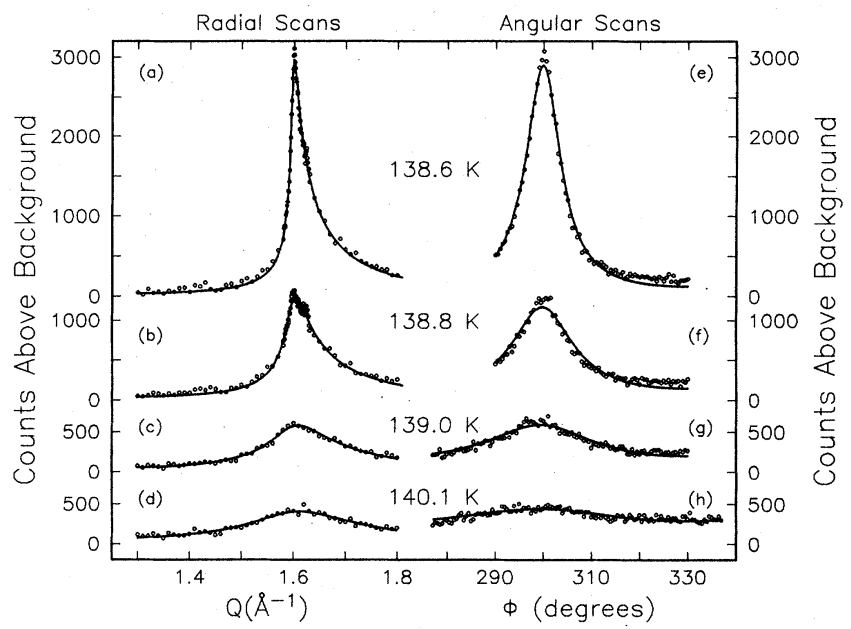

FIG. 6. Representative line-shape fits. All points are normalized to $1 \times 10^{5}$ monitor counts (although in some cases the statistics taken were as high as $1 \times 10^{6}$ counts). The measured background has been subtracted. The solid lines in the radial scans (a)-(d) are Lorentzian-spot fits. The solid lines through the angular scans $(e)-(h)$ are Lorentzian fits as described in the text.

shapes give values for the radial correlation lengths which differ by, at most, $\sqrt{3}$, that is, the ratio in half-widths of a Lorentzian and a square root of a Lorentzian with the same $\kappa$. The square-root Lorentzian shape is generated by the total mosaic average of a Lorentzian-spot profile..$^{8,9}$

Figure 5 shows a partial plot of the normalized $\chi^{2}$ for each radial fit type as a function of temperature. It is found that the power law fits best only at the lowest temperatures (as expected for the solid phase), the Lorentzian spot provides an adequate fit over all of the data, and the Lorentzian ring fits the data well at the higher tempera-

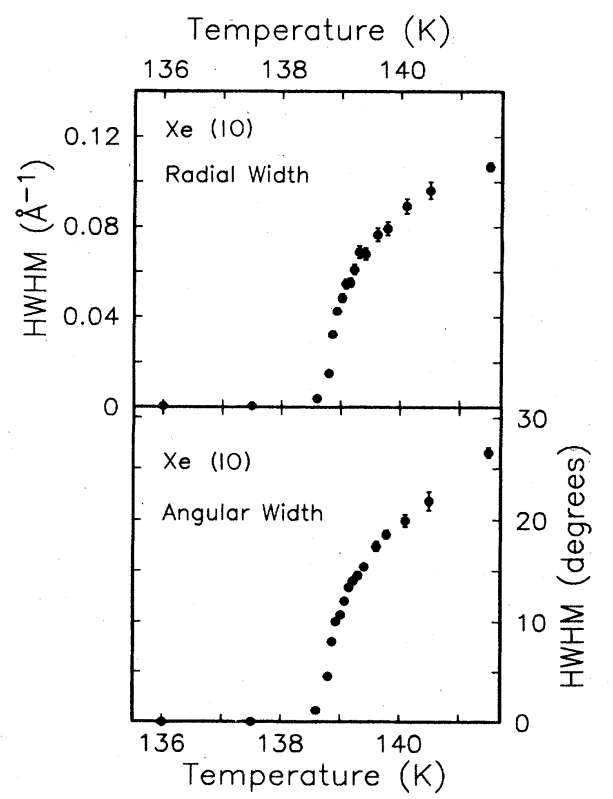

FIG. 7. Radial and angular scan HWHM as a function of temperature near $T_{M}$. The error bars represent one standard deviation as given by the weighted fits and therefore do not include systematic errors. The fits are described in the text. 


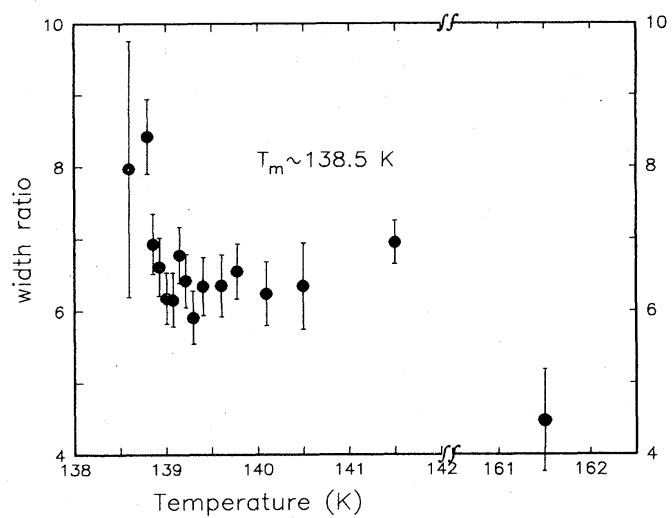

FIG. 8. The ratio of angular-to-radial width as a function of temperature. The angular widths are converted to $\AA^{-1}$ by taking the values $\Delta \theta(\mathrm{deg})$ from Fig. 7 , and setting $\Delta \theta\left(\AA^{-1}\right)=G \Delta \theta$ (radians), where $G$ is the peak position of the $\mathrm{Xe}(1,0)$ radial scans.

tures but has the lowest $\chi^{2}$ only for $T=141.5$ and $161.5 \mathrm{~K}$ (not shown). The corresponding angular scans at each temperature were fitted by a sum of three Lorentzians with the peak positions fixed at $60^{\circ}$ apart (the remaining three Lorentzians do not significantly contribute to the scattering). The Lorentzians were constrained to have the same magnitude and width so there were three adjustable parameters (the amplitude, width, and center position). This form fitted all the angular scans reasonably well; the typical scan range was over $40^{\circ}-60^{\circ}$ (see Fig. 6).

Some representative fits are shown in Fig. 6. The radial line shapes are all Lorentzian-spot fits. Figure 7 is a plot of the radial and angular widths as a function of temperature. The radial widths are from Lorentzian-spot fits, except for the point at $141.5 \mathrm{~K}$, which is from a Lorentzian-ring fit, the best fit at that temperature. The angular widths are the HWHM's of the constrained Lorentzian fits, corrected for the $3.2^{\circ}$ substrate mosaic deduced from the $\operatorname{Kr}(10)$ diffraction. It is evident that the radial and angular profiles broaden in a very similar fashion as the temperature is increased above the melting temperature of $T_{M} \sim 138.5 \mathrm{~K}$. Note that a normal $2 \mathrm{D}$ liquid should have a uniform ring of scattering with no peak in the angular scan. The finite angular widths indicate that the $2 \mathrm{D}$ solid has melted into a fluid phase with a high degree of orientational order. Although the

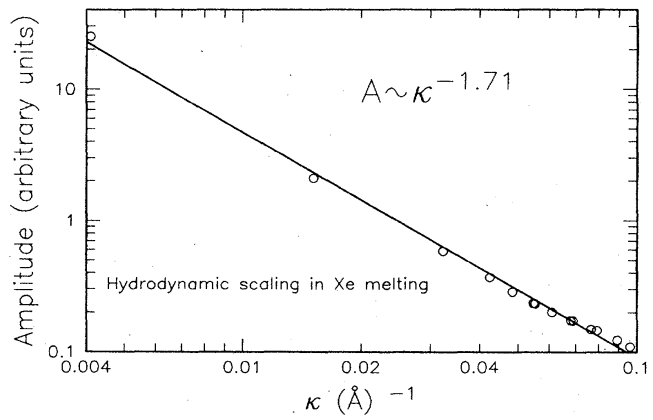

FIG. 9. Hydrodynamic scaling in the Xe liquid. The points are from Lorentzian-spot fits. The solid line shows a fitted power law corresponding to $\eta^{*}=0.29$ as described in the text.
Lorentzian-spot line shape can fit the observed radial peaks very well, it is evident that the true scattering profile is highly anisotropic. As a measure of the anisotropy, the ratios of angular to radial widths are plotted as a function of temperature in Fig. 8. The ratio is roughly constant at $\approx 6.5$ in the middle of the fluid region. More will be said about this ratio later.

Figure 7 shows that the radial correlation length $(\xi=1 / \kappa)$ grows smoothly as the temperature decreases towards the melting transition and saturates at the finitesize-limiting value imposed by the substrate. The divergence of the correlation length at a phase transition is characteristic of a continuous transition. Within the systematic limitations of this experiment imposed by the finite domain size, the $\mathrm{Xe}$ melting transition at $138.5 \mathrm{~K}$ is therefore continuous, in agreement with previous experimental results ${ }^{8,9}$ in this region of the phase diagram. Furthermore, the $\mathrm{Xe}$ melts into a fluid with substantial orientational (hexatic) order. We note that measurable short-range correlations persist at temperatures as high as $161 \mathrm{~K}$ where $\kappa \sim 0.24 \AA^{-1}$.

The amplitude and width in the fluid phase were previously found ${ }^{8,9}$ to be related by hydrodynamic scaling:

$$
A \sim \kappa^{\eta^{*}-2},
$$

where $\eta^{*}$ is the power describing the decay of correlations in the solid just below the melting temperature. Figure 9 shows a log-log plot of the peak amplitude versus $\kappa$. Fitting to a power law yields $\eta^{*}=0.29 \pm 0.03$, in close agreement with the results of experiments on $Z Y X$ graphite. On the solid side of the transition, the highest temperature at which a well-defined solid was characterized is 137.5 $\mathrm{K}$, where $\eta=0.24$. To compare this value with the hydrodynamic scaling result would require measurements in the solid phase much closer to $T_{M} \sim 138.5 \mathrm{~K}$. In practice, $\eta$ has been found to increase as $T \rightarrow T_{M}$, and $\eta$ has been estimated as $0.35 \pm 0.02$ by Dimon et al. ${ }^{9}$

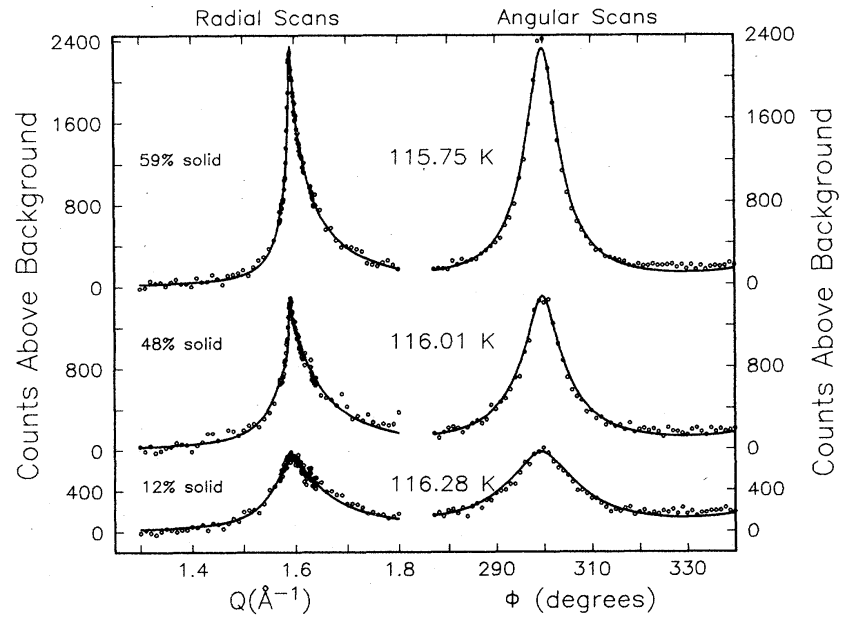

FIG. 10. Radial and angular scans in the coexistence region. The points are normalized to $1 \times 10^{5}$ monitor counts and the measured background has been subtracted. The solid lines correspond to the coexistence "variable" liquid line-shape fits as discussed in the text. The proportion of peak intensity coming from the solid component is shown in each case. 


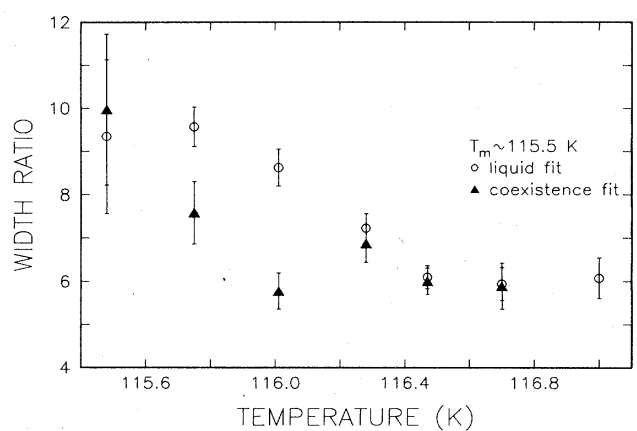

FIG. 11. The ratio of angular widths as a function of temperature in the coexistence region. The values are from the variable liquid coexistence fits (squares) or homogeneous fluid fits (circles). The angular widths were converted to $\AA^{-1}$ as Fig. 5 .

A small set of scans at eight temperatures from 115 to $117 \mathrm{~K}$ was made to examine the melting transition in a region where it is believed to be first order. ${ }^{9}$ Of these, the scan at $115 \mathrm{~K}$ was clearly seen to be in the solid phase and described by a power-law line shape. The other scans were fitted as Lorentzian spots and also to a composite line shape in order to check for liquid-solid coexistence.

Previous $x$-ray scattering investigations of Xe melting in the coexistence region were carried out as a function of pressure with the temperature held constant. Under these circumstances, the line shape is uniquely determined as a sum of contributions from the pure liquid and pure solid line shapes at the phase boundaries. In the present experiment the temperature was varied while stepping through the coexistence region, so the correlation length of the fluid state at the appropriate phase boundary can vary at each point.

The coexistence fits were therefore carried out by two methods. In the first the scans were assumed to be the sum of a solid peak with a line shape identical to that measured at $115 \mathrm{~K}$ and a Lorentzian-spot component. The radial and angular scans were fitted simultaneously, with six parameters allowed to vary: the ratio of solidto-liquid intensity, the peak positions in radial and angular scans, the widths of radial and angular scans, and an overall scaling factor. The second method was identical to the first, except that the widths were fixed at the values obtained by a pure liquid fit at a postulated phase boundary.

In practice, the fluid fits and "variable liquid" coexistence fits were found to describe the scattering nearly equally well, although the coexistence fits were somewhat better at temperatures below $116.28 \mathrm{~K}$. The fixed "liquid" coexistence model was unable to fit the data well. Some typical data fitted by the variable liquid coexistence model are shown in Fig. 10.

The ratio of angular and radial widths in the fluid, or fluid part of the coexisting phases, are shown in Fig. 11. The data show a good deal of scatter, but the width ratios are essentially consistent with those measured at $138 \mathrm{~K}$.

\section{DISCUSSION}

The structure factor for a normally disordered liquid should be an isotropic ring of scattering. From our re- sults it is therefore apparent that the Xe monolayer melts into an orientationally ordered (hexatic) fluid in the continuous melting regoin $(138 \mathrm{~K})$ and probably also at 116 K. We now discuss the possible origins of the observed orientational order.

We reiterate that the substrate can affect the order of the transition as well as the amount of orientational order. With a finite orientational field $h_{6}$ the formation or orientationally unaligned blocks, and hence of grain boundaries, is inhibited. Crudely, taking $h_{6}$ as an orientational energy per unit area [see Eq. (10)], and $\xi_{6}^{0}$ as the largest substrate-free orientational correlation length, the fluctuations leading to first-order melting can be suppressed when ${ }^{10,31}$

$$
h_{6}\left(\xi_{6}^{0}\right)^{2} \geq k_{B} T_{M} .
$$

If the substrate-free melting transition is weakly first order, $\xi_{6}^{0}$ will be large and an extremely small $h_{6}$ will be sufficient to make the transition continuous. We conclude, therefore, that no experiment on a substrate can show that the transition would be continuous in the absence of a substrate; one can argue that if $h_{6}$ is small, $\xi_{6}^{0}$ must be large for the transition to be continuous.

From the behavior of the orientational correlations, one can, however, learn about the nature of the fluid phase; we note that in our experiment the orientational correlation function, and hence $\xi_{6}$, cannot be measured directly. Even so, it is possible to measure the mean-square fluctuations in the angular order, $\left\langle\delta \theta^{2}\right\rangle$. To extract $\left\langle\delta \theta^{2}\right\rangle$ from our data, we take the view that angular fluctuations induce angular disorder that behaves exactly as if the crystal had a larger mosaic spread. We assume that the diffuse scattering about each reciprocal-lattice point $\mathbf{G}$ is intrinsically a Lorentzian of the form

$$
S(\mathbf{Q}) \sim\left(|\mathbf{Q}-\mathbf{G}|^{2}+\kappa^{2}\right)^{-1} .
$$

With a perfect crystal, an angular scan through such a spot would have a half-width at half maximum given by

$$
\Delta \theta_{r}=2 \sin ^{-1}\left(\frac{\kappa}{2 G}\right) .
$$

The substrate mosaic spread $\Delta \theta_{s}$ is a fixed and known quantity (see Fig. 3). The angular fluctuations can be re-

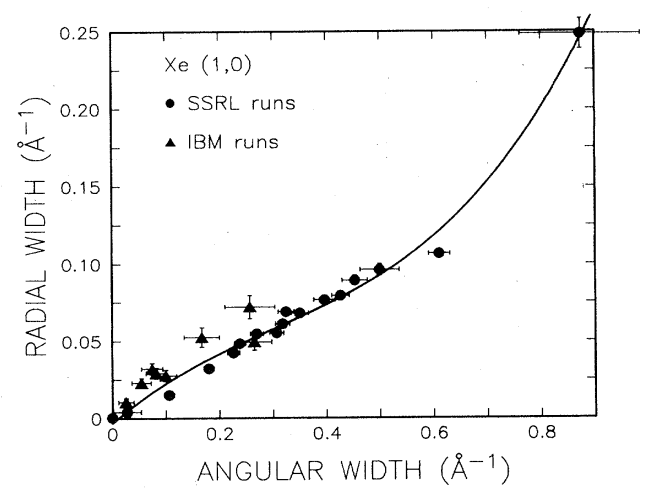

FIG. 12. Radial width versus excess angular width. The excess angular widths are determined as discussed in the text. The solid line is a guide to the eye. 
lated to the measured half-widths $\Delta \theta_{m}$ of our angular scans by

$$
\left(\left\langle\delta \theta^{2}\right\rangle\right)^{1 / 2}=\Delta \theta_{m}-\Delta \theta_{s}-\Delta \theta_{r},
$$

where the linear addition rule is assumed since the measured profiles are Lorentzians. These results can be converted to inverse angstroms by defining $\kappa_{\theta} \equiv G\left(\left\langle\delta \theta^{2}\right\rangle\right)^{1 / 2}$.

In Fig. 12 we plot $\kappa$ versus $\kappa_{\theta}$. The circles represent the results of the present experiment; triangles are results from an earlier experiment at IBM using a rotating anode. ${ }^{2}$ When the correlation lengths are long we have $\kappa \propto \kappa_{\theta}$; only at much higher temperatures $(T \sim 160 \mathrm{~K})$ does this linearity appear to break down.

To aid in the interpretation of these data we consider the following method of calculating $\left\langle\delta \theta^{2}\right\rangle$. The Hamiltonian for a hexatic phase ${ }^{10}$ can be written

$$
\mathscr{H}=\frac{K_{A}}{2} \int(\nabla \theta)^{2} d^{2} r+\frac{1}{2} \int h_{6} \theta^{2} d^{2} r
$$

in the continuum elastic approximation. In Eq. (10) $\theta(r)$ is the field of local $\mathrm{Xe}-\mathrm{Xe}$ bond orientations, $K_{A}$ is a Franck constant describing bond-angle stiffness, and $\frac{1}{2} h_{6} \theta^{2}$ is the energy cost per unit area to rotate the xenon layer by an angle $\theta$ with respect to the substrate. Since the Hamiltonian in Eq. (10) is harmonic, it is straightforward to compute the mean-square angular fluctuations $10,12,14$

$$
\left\langle\delta \theta^{2}\right\rangle=\frac{k_{B} T}{2 \pi} \int_{2 \pi / L}^{q_{c}} \frac{q d q}{K_{A} q^{2}+h_{6}} .
$$

The uniform distortion term ${ }^{2} k_{B} T / h_{6} L^{2}$ has been assumed to vanish because the overlayer is not free to rotate uniformly with respect to the substrate due to orientational pinning at the boundary. ${ }^{32}$ The cutoff wave vector $q_{c}$ is $(2 / \pi \sqrt{3})^{1 / 2}(2 \pi / a)$ for a hexagonal lattice of lattice constant $a$ and the size of the system is taken as $L \times L$. The fact that $K_{A}=\infty$ in the solid phase can be accounted for by setting

$$
q_{c}=(2 / \pi \sqrt{3})^{1 / 2}(2 \pi / \xi) .
$$

The validity of Eq. (11) in conjunction with Eqs. (7) and (9) will be discussed later. The integral (11) yields

$$
\left\langle\delta \theta^{2}\right\rangle=\frac{k_{B} T}{4 \pi K_{A}} \ln \left[\frac{K_{A} q_{c}^{2}+h_{6}}{K_{A}(2 \pi / L)^{2}+h_{6}}\right] .
$$

In a well-correlated hexatic phase, $K_{A} \sim E_{c} \xi^{2}$, where $E_{c}$ is the core energy of a dislocation. ${ }^{10}$ Therefore, as $\xi \rightarrow \infty$, $\left\langle\delta \theta^{2}\right\rangle \sim k_{B} T / K_{A}$, and one expects $\kappa=1 / \xi$ to be proportional to $\kappa_{\theta}$ as observed experimentally.

Conversely, at the transition from hexatic to liquid, $K_{A}$ should jump to zero from a universal value of $72 k_{B} T / \pi$. Therefore, in the limit of a tiny $h_{6}$ the $\kappa$ versus $\kappa \theta$ plot should show a pronounced bend (see Fig. 13). On the other hand, a finite $h_{6}$ will limit the rapid growth of $\kappa_{\theta}$. The heuristic dependence of the angular and radial widths is shown in Fig. 13.

Figures 8 and 12 show that as $\kappa \rightarrow 0$ there is an approximately linear dependence of $\kappa$ on $\kappa_{\theta}$. Because of the possible systematic errors, we do not believe that the points in

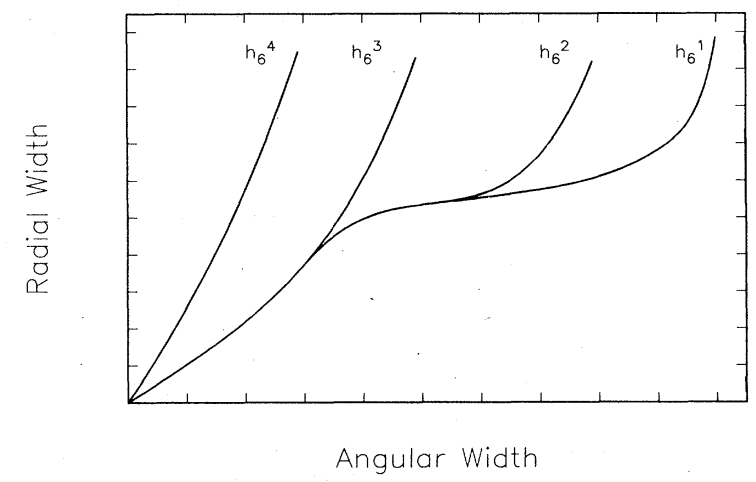

FIG. 13. Schematic of the heuristic dependence of the radial spot with $\kappa$ versus the angular spot width $\kappa_{\theta}$ for various values of the substrate field $h_{6}, h_{6}^{4}>h_{6}^{3}>h_{6}^{2}>h_{6}^{1}$.

Fig. 8 at small $\kappa$ rule out a constant ratio of $K$ to $K_{\theta}$. These results are consistent with that expected for a hexactic phase with small $h_{6}$. However, it is clear that there is no evidence of the jump in $K_{A}$ suggested in Ref. 2 . This implies that the orientational field is sufficient to smear out any hexatic-to-liquid transition.

In the limit where $(2 \pi / L)^{2} K_{A} \gg h_{6}$, and assuming $K_{A}=2 E_{c}(\xi / a)^{2},{ }^{10}$ one can estimate the value of the $E_{c}$ from Eq. (12). The resulting dislocation core energy, $E_{c} \sim k_{B} T_{M}$, is of the same order as the value at which Saito finds a change from first order to continuous melting $^{23}$ in a dislocation array simulation, and also where Chui finds that grain-boundary-driven melting switches from strongly to weakly first order. ${ }^{18}$ The value of $E_{c}$ for the $2 \mathrm{D}$ electron lattice is known to be about $50 \%$ larger than Saito's value for continuous melting. ${ }^{32}$ For the electron system, Morf ${ }^{21}$ has found that melting is continuous, in agreement with the KTHNY theory.

The approximations leading to Eqs. (7), (9), and (11) are numerous. Equation (10) is strictly a harmonic ("spinwave-like") approximation which should be valid in the hexatic phase if disclination pairs renormalize out of the problem. ${ }^{10}$ The quadratic $q$ dependence of $|\delta \theta(q)|^{2}$ [Eq. (11)] should be valid for small $q$ only, and the expilict dependence of $\left\langle\delta \theta^{2}\right\rangle$ on the upper momentum cutoff $q_{c}$ is artificial. Furthermore, the extraction of $\left\langle\delta \theta^{2}\right\rangle$ from the measured $S(Q)$ is not rigorous. Aeppli and Bruinsma ${ }^{33}$ have carried out a calculation of the angular dependence of $S(Q)$ for $T$ near $T_{H M}$. They find that at high temperatures $S(Q)$ is well approximated by a sixfold sinusoidal modulation of a liquid ring. At lower temperatures ( $T$ near $T_{M}$ ), a form with six Lorentzian spots is a better approximation. We have found empirically that six Lorentzian spots fit the data well over the entire temperature range. At high temperatures, the fitted width of the Lorentzians becomes comparable with their $60^{\circ}$ separation, and the resulting scattering is not fundamentally different from a modulated liquid ring; at $161 \mathrm{~K}$ the number of counts above background in the angular scans varies by only about $15 \%$. Therefore, the use of six Lorentzian spots allows for a simple parametrization of the angular data in terms of a single parameter $\kappa_{\theta}$ over the entire data range. However, in spite of these approximations, the KTHNY theory with $h_{6}=0$ gives a good description of 
the data for $T$ near $T_{M}$. Indeed, the fact that the core energy estimated above for xenon melting near its tricritical point is comparable with Saito's tricritical value of the dislocation core energy is a remarkable success of the theory. On the other hand, it is clear that $h_{6}$ is sufficiently large to smear out the hexatic-isotropic fluid transition.

Crudely, the condition that $h_{6}$ be neglected in Eq. (11) is $h_{6}<K_{A}(2 \pi / L)^{2}$, which for $T$ near $T_{M}$ becomes $h_{6}<4 \pi^{2} E_{c}(\xi / L)^{2}$. This condition is easily satisfied for $T$ near $T_{M}$. However, as $T$ increases, $\xi$ decreases, and $h_{6}$ becomes progressively important, until for $T \geq T_{H M}$ an arbitrarily small $h_{6}$ will dominate. ${ }^{33}$

We have argued above that xenon melting at $138 \mathrm{~K}$ is consistent with the KTHNY hexatic picture of 2D melting, with $h_{6}$ effects being small for $T$ near $T_{M}$. We now consider alternate models. In particular, we consider the possibility that the same results can be produced by an isotropic liquid with orientational order induced solely by the substrate through $h_{6}$. It is unfortunately very difficuit to estimate the effects of the substrate reliably. The value of $h_{6}$ is not well known; there are no estimates in the literature of $h_{6}$ in the fluid phase and only limited information for the solid phase.

The orientational epitaxy potentials of Novaco and McTague ${ }^{3,34}$ cannot be used in a simple way since they predict that a xenon monolayer will rotate with respect to the graphite $R 30^{\circ}$ direction, whereas, in fact, at these temperatures the xenon does not rotate. Thus, orientational epitaxy potentials have a maximum at the $R 30^{\circ}$ direction, whereas Eq. (10) has a quadratic minimum. For this reason, estimates of $h_{6}$ based on the Novaco-McTague potentials for $\mathrm{Xe}$ are somewhat ambiguous. One can fit these potentials to Eq. (10) by ignoring the local structure at $\theta=0$. Not surprisingly, the resulting values of $h_{6}$ depend strongly on the region of fit. Furthermore, the Xegraphite potentials are uncertain to about a factor of $2 .{ }^{35,36}$ Various approximations of this type yield $T=0$ values for $h_{6}^{0} / k_{B}$ between 1 and $20 \mathrm{~K}$. Here, we use $h_{6}^{0}$ to represent the orientational field per atom as compared with $h_{6}$ which is the field per unit area. The above estimates are for $T=0$. It is generally expected that $h_{6}$ should be renormalized downward for $T>0 .{ }^{3,34}$ This occurs because the thermal disorder of the adsorbed layer prevents it from taking maximum advantage of a specific orientation. Unfortunately, the magnitude of this effect is not known, and, indeed, the value of $h_{6}$ for a liquid is even more uncertain. ${ }^{3}$

The orientational epitaxy potential of $\operatorname{argon}^{3}$ suggests that an argon monolayer should have an $h_{6}$ weaker than or equal to that of xenon. However, in practice the argon layers show more substrate effects, ${ }^{37,38}$ and Fain ${ }^{38}$ has suggested that domain-wall effects ${ }^{39}$ may in some way be the primary cause of the orientational order. It is clear that the effects of the substrate on fluid orientational order are not well understood.

With these limitations in mind, we can still attempt to estimate the effect of the substrate on a simple Xe liquid monolayer. Equation (10) should be valid for a normal liquid, except that in a normal liquid there is no bondangle stiffness, so one would expect the Franck constant $K_{A}$ to vanish as $q \rightarrow 0$, whereas the KTHNY hexatic has
$K_{A}$ finite at $q=0$. When the system is a well-correlated fluid, however, there will be a residual stiffness at finite $q$. The most conservative approximation is to assume that for a well-correlated fluid all fluctuations in the angular order are on length scales longer than $\xi_{6}$, and there is infinite orientational stiffness on length scales less than $\xi_{6}$. Then,

$$
\left\langle\delta \theta^{2}\right\rangle \approx \frac{k_{B} T}{2 \pi} \int_{2 \pi / L}^{\bar{q}} \frac{q d q}{h_{6}},
$$

where we choose

$$
\bar{q}=(2 / \pi \sqrt{3})^{1 / 2}\left(2 \pi / \xi_{6}\right) .
$$

Setting $\kappa_{\theta}^{2}=|G|^{2}\left\langle\delta \theta^{2}\right\rangle$, after some algebra the result leads to

$$
\kappa_{\theta}^{2}=\left(\frac{16 \pi^{2}}{3}\right) \frac{k_{B} T}{h_{6}^{0} \xi_{6}^{2}},
$$

where $h_{6}^{0}$ is an energy per atom. Equation (14) leads to the condition

$$
h_{6}^{0}\left(\xi_{6} / \xi\right)^{2}=\left(16 \pi^{2} / 3\right)\left(\kappa / \kappa_{\theta}\right)^{2} k_{B} T,
$$

which for our data implies that

$$
h_{6}^{0}\left(\xi_{6} / \xi\right)^{2} \sim 3 k_{B} T_{M}
$$

will explain the observed orientational order with $\xi_{6} / \xi$ constant. The numerical factor in Eq. (16) is quite uncertain. Given this caveat, it is clear that, within this model, the Xe data can be explained either if the field $h_{6}^{0}$ is much greater than that estimated above, which seems unlikely, or if the orientational correlation length, which we cannot measure, is much greater than the positional correlation length.

In principle, the above calculation can be extended to account more correctly for the wave-vector dependence of $K_{A}$. The bond-angle stiffness can be treated in the Debye-Hückel approximation ${ }^{14}$ in which

$$
K_{A}(q)=K_{0}\left[1+\left(\frac{1}{q \xi_{6}}\right]^{2}\right]^{-1},
$$

where $K_{0}$ is a "bare" Franck constant. The orientational fluctuations are then calculated by substituting Eq. (17) into Eq. (11). However, physically, the usual DebyeHückel form does not allow for the possibility that there are two important length scales in the problem. If one assumes that the xenon fluid is a liquid with $\xi_{6}>\xi$, then on length scales between $\xi_{6}$ and $\xi$ the system behaves like a hexatic. One might then expect

$$
K_{A} \sim 2 E_{c} \xi^{2}\left[1+\left(\frac{1}{q \xi_{6}}\right)^{2}\right]^{-1},
$$

which leads to $\kappa_{\theta} \propto \kappa_{r}$ as observed experimentally. The condition that this describes the data for $T$ near $T_{M}$ approximately reduces to Eq. (16) from the sharp-cutoff model. 


\section{SUMMARY}

The melting of $\mathrm{Xe}$ monolayers adsorbed on single crystals of exfoliated graphite has been investigated using high-resolution synchrotron $\mathrm{x}$-ray scattering. The solid melts into an orientationally ordered fluid at $138 \mathrm{~K}$, and at $116 \mathrm{~K}$ there is some evidence of coexistence of a solid with an orientationally ordered fluid. No experiment can prove with certainty that a transition is continuous. It can, however, set limits on the size of the first-order jump by setting lower bounds on the positional correlations of the high-temperature (fluid) phase. In the present case we can argue that $\xi$ reaches at least about $100 \AA$. Furthermore, in the liquid phase, at $138 \mathrm{~K}$ the behavior of the orientational and radial order is consistent with the hexatic picture of the melting transition; a substrate field of a few degrees Kelvin is sufficient to smear out any hexaticto-liquid transition. The value of the dislocation core energy needed to fit the data is comparable to the tricritical value at which Saito finds a crossover from first-order to continuous melting. ${ }^{19}$ The data indicate much stronger orientational order than would be given by current estimates of the graphite substrate field. We cannot rule out an alternate, artificial model for melting in which there is a continuous melting transition at which $\xi_{6}, \xi \rightarrow \infty$, $\xi_{6} / \xi \rightarrow$ const, and $\left(\xi_{6} / \xi\right)^{2} h_{6}^{0} \sim 3 k_{B} T_{M}$. It is unambiguous, however, that $\xi_{6}$ becomes extremely large if not macroscopic in the liquid phase. Our understanding of substrate effects must be improved in order to make a more definitive statement.

\section{ACKNOWLEDGMENTS}

It is a pleasure to acknowledge valuable discussions and exchange of information with R. Bruinsma, C. Herring, P. Dimon, S. Fain, G. Grinstein, D. Nelson, E. D. Specht, and A. Novaco. We would also like to thank H. Homma for the preparation of intercalate graphite samples. This work was partially done at the Stanford Synchrotron Radiation Laboratory which is supported by the U.S. Department of Energy Office of Basic Energy Sciences. One of us (S.E.N.) was supported by the Natural Sciences and Engineering Research Council of Canada. T.F.R. was supported by the Materials Research Laboratory Program of the National Science Foundation at the University of Chicago under Grant No. 82-16892. The MIT collaborators were supported by the Joint Services Electronics Program under Contract No. DAAG-29-83-K0003 and by the National Science Foundation Materials Research Laboratory under Contract No. DMR-81-19295. R.C. was supported by National Science Foundation Low Temperature Physics Grant No. DMR-84-04975. Work at Brookhaven National Laboratory was supported by the U. S. Department of Energy under Contract No. DE-AC0276-CH00016.
*Permanent address: Department of Physics, University of Florida, 215 Williamson Hall, Gainsville, FLA 32611.

${ }^{1}$ R. Clarke, P. M. Horn, S. E. Nagler, and T. F. Rosenbaum, J. Appl. Phys. 55, 1231 (1984).

${ }^{2}$ T. F. Rosenbaum, S. E. Nagler, P. M. Horn, and R. Clarke, Phys. Rev. Lett. 50, 1791 (1983).

${ }^{3}$ A. D. Novaco and J. P. McTague, Phys. Rev. Lett. 38, 1286 (1977); J. P. McTague and A. D. Novaco, Phys. Rev. B 19, 5299 (1979).

${ }^{4}$ A. Thomy and X. Duval, J. Chim. Phys. Phys. Chim. Biol. 67, 1101 (1970).

5J. Morrison and J. J. Lander, Surf. Sci. 5, 163 (1966); 6, 1 (1967).

${ }^{6}$ H. Taub, K. Carniero, J. K. Kjems, L. Passell, and J. P. McTague, Phys. Rev. B 16, 4551 (1977).

${ }^{7}$ R. J. Birgeneau, E. M. Hammonds, P. Heiney, P. W. Stephens, and P. M. Horn, in Ordering in Two Dimensions, edited by S. K. Sinha (Elseiver/North-Holland, New York, 1980), p. 29; M. Nielsen, J. Bohr, K. Kjaer, and J. Als-Nielsen, Nucl. Instrum. Methods 208, 549 (1983).

8P. A. Heiney, P. W. Stephens, R. J. Birgeneau, P. M. Horn, and D. E. Moncton, Phys. Rev. B 28, 6416 (1983).

${ }^{9}$ P. Dimon, P. M. Horn, M. Sutton, R. J. Birgeneau, and D. E. Moncton, Phys. Rev. B 31, 437 (1985).

${ }^{10}$ B. I. Halperin and D. R. Nelson, Phys. Rev. Lett. 41, 121 (1978); 41, 519 (1978); D. R. Nelson and B. I. Haperin, Phys. Rev. B 19, 2457 (1979).

${ }^{11}$ A. P. Young Phys. Rev. B 19, 1855 (1979).

12J. M. Kosterlitz and D. J. Thouless, J. Phys. C 5, L124 (1972); 6, 1181 (1973).

${ }^{13}$ V. L. Berezinksii, Zh. Eksp. Teor. Fiz. 61, 64 (1971) [Sov. Phys._JETP 34, 34 (1971)].
${ }^{14}$ D. R. Nelson, in Fundamental Problems in Statistical Mechanics $V$, edited by E. G. D. Cohen (North-Holland, New York, 1980).

${ }^{15}$ D. R. Nelson, Phys. Rev. B 26, 269 (1982).

${ }^{16}$ K. J. Strandburg, S. A. Solla, and G. V. Chester, Phys. Rev. B 28, 27.18 (1983).

${ }^{17}$ G. Grinstein and J. Toner, Phys. Rev. Lett. 51, 2386 (1983).

${ }^{18}$ S. T. Chui, Phys. Rev. Lett. 48, 935 (1982); Phys. Rev. B 28, 1978 (1983).

${ }^{19}$ H. Kleinert, Phys. Lett. 95A, 381 (1983); S. Ami and H. Kleinert, J. Phys. (Paris) Lett. 45, L-877 (1984).

${ }^{20}$ V. I. Marchenko, Pis'ma Zh. Eksp. Teor. Fiz. 33, 397 (1981) [JETP Lett. 33, 381 (1981)].

${ }^{21}$ R. H. Morf, Helv. Phys. Acta 56, 743 (1983).

${ }^{22}$ F. F. Abraham, Phys. Rev. Lett. 50, 978 (1983); S. W. Koch and F. F. Abraham, Phys. Rev. B 27, 2964 (1983).

${ }^{23}$ Y. Saito, Phys. Rev. Lett. 48, 1114 (1982); Phys. Rev. B 26, 6239 (1982).

${ }^{24}$ R. H. Morf, in Physics of Intercalculation Compounds, Vol. 38 of Springer Series in Solid State Sciences, edited by L. Pietronero and E. Tosatti (Springer, Berlin, 1981), p. 252.

${ }^{25}$ P. A. Heiney, Ph.D. thesis, Massachusetts Institute of Technology, 1982; P. Dimon, Ph.D. thesis, University of Chicago, 1984.

${ }^{26}$ D. E. Moncton and G. S. Brown, Nucl. Instrum. Methods 208, 579 (1983).

${ }^{27}$ P. W. Stephens, P. A. Heiney, R. J. Birgeneau, P. M. Horn, D. E. Moncton, and G. S. Brown, Phys. Rev. B 29, 3512 (1984).

${ }^{28}$ Y. Imry and L. Gunther, Phys. Rev. B 3, 3939 (1971).

${ }^{29}$ P. Dutta and S. K. Sinha, Phys. Rev. Lett. 47, 50 (1981).

${ }^{30}$ D. A. Huse, Phys. Rev. B 28, 6110 (1983).

${ }^{31}$ D. R. Nelson (private communication). 
32D. S. Fisher, B. I. Halperin, and R. Morf, Phys. Rev. B 20, 4692 (1979).

${ }^{33}$ G. Aeppli and R. Bruinsma, Phys. Rev. Lett. 53, 2133 (1984).

${ }^{34}$ A. D. Novaco (private communication).

${ }^{35}$ G. Vidali and M. W. Cole, Phys. Rev. B 29, 6736 (1984).

${ }^{36}$ K. L. D'Amico, A. E. Moncton, E. D. Specht, R. J. Bir- geneau, S. E. Nagler, and P. M. Horn (unpublished).

37J. A. McTague, J. Als-Nielsen, M. Nielsen, and J. Bohr, Phys. Rev. B 25, 7765 (1982).

${ }^{38}$ C. G. Shaw, S. C. Fain, and M. D. Chinn, Phys. Rev. Lett. 41, 955 (1978); S. C. Fain (private communication).

${ }^{39}$ H. Shiba, J. Phys. Soc. Jpn. 46, 1852 (1979); 48, 211 (1980). 